\title{
Unraveling a concealed resonance by multiple Kondo transitions in a quantum dot
}

\author{
Aritra Lahiri, ${ }^{1,2}$ Tokuro Hata, ${ }^{3}$ Sergey Smirnov, ${ }^{4}$ Meydi Ferrier, ${ }^{3,5}$ Tomonori Arakawa, ${ }^{3}$ Michael Niklas, ${ }^{1}$ \\ Magdalena Marganska, ${ }^{1}$ Kensuke Kobayashi, ${ }^{3,6,7,{ }^{*}}$ and Milena Grifoni ${ }^{1, \dagger}$ \\ ${ }^{1}$ Institut für Theoretische Physik, Universität Regensburg, D-93040 Regensburg, Germany \\ ${ }^{2}$ School of Physics and Astronomy, University of Minnesota, Minneapolis, Minnesota 55455, USA \\ ${ }^{3}$ Department of Physics, Graduate School of Science, Osaka University, 560-0043 Osaka, Japan \\ ${ }^{4}$ P. N. Lebedev Physical Institute of the Russian Academy of Sciences, 119991 Moscow, Russia \\ ${ }^{5}$ Laboratoire de Physique des Solides, CNRS, Univ. Paris-Sud, Université Paris Saclay, 91405 Orsay Cedex, France \\ ${ }^{6}$ Center for Spintronics Research Network (CSRN), Graduate School of Engineering Science, Osaka University, Osaka 560-8531, Japan \\ ${ }^{7}$ Institute for Physics of Intelligence and Department of Physics, The University of Tokyo, Tokyo 113-0033, Japan
}

(Received 10 August 2019; revised manuscript received 26 November 2019; published 6 January 2020)

\begin{abstract}
Kondo correlations are responsible for the emergence of a zero-bias peak in the low temperature differential conductance of Coulomb blockaded quantum dots. In the presence of a global SU(2) $\otimes S U(2)$ symmetry, which can be realized in carbon nanotubes, they also inhibit inelastic transitions which preserve the Kramers pseudospins associated to the symmetry. We report on magnetotransport experiments on a Kondo correlated carbon nanotube where resonant features at the bias corresponding to the pseudospin-preserving transitions are observed. We attribute this effect to a simultaneous enhancement of pseudospin-nonpreserving transitions occurring at that bias. This process is boosted by asymmetric tunneling couplings of the two Kramers doublets to the leads and by asymmetries in the potential drops at the leads. Hence, the present work discloses a fundamental microscopic mechanisms ruling transport in Kondo systems far from equilibrium.
\end{abstract}

DOI: 10.1103/PhysRevB.101.041102

The Kondo effect [1], a quintessential example of strong correlations in a many-body system, describes the screening of a localized spin by a Fermi sea of conduction electrons. Quantum dots (QDs) with odd electron occupancy behaving as effective spin-1/2 systems provide a simple realization of the SU(2) Kondo effect. Here the entanglement of the dot spin with the lead conduction electrons leads to the emergence of a zero-bias peak in the low temperature differential conductance $[2,3]$. The Kondo effect in QDs also has more exotic realizations, provided that the associated degrees of freedom are conserved during tunneling. A prominent example is carbon nanotube (CNT) QDs where the presence of orbital (valley) and spin degrees of freedom leads to the SU(4) [4-9] and the $S U(2) \otimes S U(2)$ Kondo effects [10-13]. The latter occurs when the valley and spin degeneracy of a CNT longitudinal mode is broken by spin-orbit coupling [14] or valley mixing [15]. Time-reversal symmetry results in two Kramers doublets separated by an inter-Kramers splitting $\Delta$, as seen in Fig. 1(a). A signature of the $S U(2) \otimes S U(2)$ Kondo effect is thus two inelastic peaks, symmetrically located with respect to a zero-bias peak, in a CNT with single electron or single hole occupancy [12]. Such features are seen in our experiment, as shown in Figs. 1(b) and 1(c).

In analogy to the more conventional $\mathrm{SU}(2)$ case, a pseudospin can be associated to each Kramers doublet of the CNT and to the lead electrons [15]. As in the SU(2) case [16], tunneling results in an effective antiferromagnetic exchange

\footnotetext{
*kensuke@meso.phys.sci.osaka-u.ac.jp

†milena.grifoni@physik.uni-regensburg.de
}

coupling among the CNT and lead pseudospins [15,17]. In the Kondo regime this favors virtual tunneling transitions across the QD where the pseudospin of the CNT gets flipped. While the central peak accounts for quasielastic pseudospin-flip transitions within the same Kramers doublet ( $\mathcal{T}$ transition), the inelastic peaks involve one state in the lower and one in the upper Kramers doublet. One distinguishes between chiral $(C)$ and particle-hole $(P)$ transitions if the two states involve the opposite or the same pseudospin, respectively [see Fig. 2(a)]. Kondo correlations inhibit transitions of the $P$ type, as confirmed by transport experiments at low magnetic fields [4,6,11-13]. However, $P$ transitions can be observed in the weak-coupling regime, where Kondo exchange is not important and only lowest order cotunneling processes are responsible for the inelastic peaks [13,18-20].

In this Rapid Communication, we demonstrate experimentally the puzzling emergence of a resonance at energies of the inelastic $P$ transition in Kondo-correlated CNT QDs. Noticeably, the $P$ resonance is clearly seen only for a given bias polarity, suggesting its association with lead coupling asymmetries. We present a comprehensive theoretical analysis based on the Keldysh effective action (KEA) theory [12,21], addressing the role of asymmetries in Kondo-correlated CNT QDs. The $P$-like features arise from the coherent addition of a $C$ - and a $\mathcal{T}$-type transition, which occurs when the applied bias equals the energy of the inelastic $P$ resonance, becoming relevant for different couplings of the Kramers doublets to the leads.

Experimental results. Our device is made of a CNT grown by chemical vapor deposition and connected to $\operatorname{Pd}(6 \mathrm{~nm}) / \mathrm{Al}(70 \mathrm{~nm})$ leads. Fabrication details can be found 
(a)
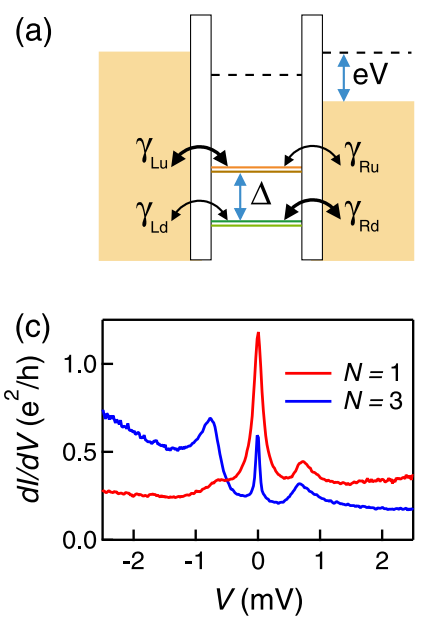

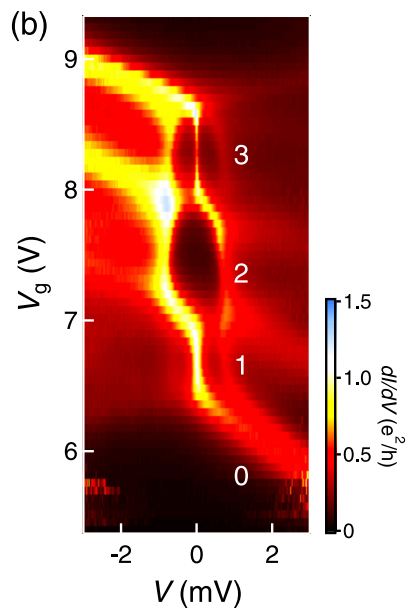

FIG. 1. The $S U(2) \otimes S U(2)$ Kondo effect. (a) A CNT longitudinal shell possesses two Kramers doublets separated by a splitting $\Delta$. The upper (u) and lower (d) doublets are coupled to left $(L)$ and right $(R)$ leads by tunneling couplings $\gamma_{L p}$ and $\gamma_{R p}$, where $p=\mathrm{u}, \mathrm{d}$. (b) Experimental differential conductance as a function of gate, $V_{\mathrm{g}}$, and bias, $V$, voltages for different occupation of a longitudinal shell. In the valleys with odd occupancy a zero-bias Kondo ridge is clearly seen. Two additional ridges, symmetrically located with respect to the central Kondo peak, are observed at finite bias. The asymmetric response implies the presence of asymmetries in the problem. (c) Bias traces taken at gate voltages corresponding to the center of the $N=1$ and $N=3$ valleys show inelastic peaks with roughly the same spacing $\Delta$.

in Ref. [8]. The differential conductance of our CNT QD is shown in Fig. 1(b) as a function of the applied bias $V$ and gate voltage $V_{\mathrm{g}}$. A small perpendicular magnetic field $B=0.02 \mathrm{~T}$ is applied to suppress superconductivity of $\mathrm{Al}$ in the leads. A Kondo ridge, corresponding to the yellow line at zero bias, is recognized in the Coulomb valleys with occupation $N=1$ and $N=3$ of a longitudinal shell. In the $N=0,2$ valleys no Kondo ridge is seen. Additional inelastic peaks, symmetrically located with respect to zero bias, are observed for the $N=1,2,3$ valleys. Bias traces taken at gate voltages corresponding to the center of a valley are shown in Fig. 1(c). From such traces a Kramers splitting of $\Delta \simeq 0.7 \mathrm{meV}$ is estimated. From the width of the zero-bias peaks Kondo temperatures of $T_{K 1}=1 \mathrm{~K}$ and $T_{K 3}=0.37 \mathrm{~K}$ are extracted for valley $N=1$ and $N=3$, respectively. Since our experiments are taken at temperatures around $T=30 \mathrm{mK}$, it holds $T<T_{K}$. Furthermore, from the evolution in perpendicular magnetic field [see Eq. (2) below], we can extract a spin-orbit coupling splitting $\Delta_{\mathrm{SO}}=0.07 \mathrm{meV}$ and a larger valley mixing energy $\Delta_{\mathrm{K}, \mathrm{K}^{\prime}}=0.7 \mathrm{meV}$ for both Kondo valleys.

A magnetic field breaks time-reversal symmetry and hence also Kramers degeneracy. The expected evolution of the single-particle energy spectrum of a longitudinal shell and its associated excitation spectrum are shown in Figs. 2(a) and 2(b). Notice that $C$ excitations are independent of the magnetic field until the anticrossing of the inner levels $(2,3)$. The Kondo effect is, however, a many-body phenomenon, and differences in the excitation spectrum are expected. The experimental magnetoconductance is shown in Figs. 2(c) and

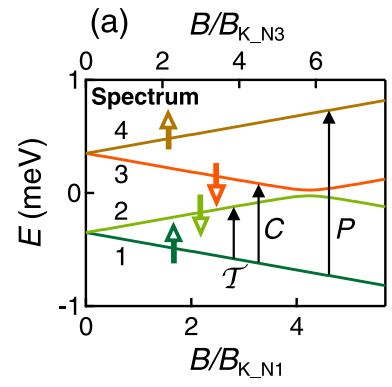

(b) $B / B_{\mathrm{K}_{-} \mathrm{N} 3}$
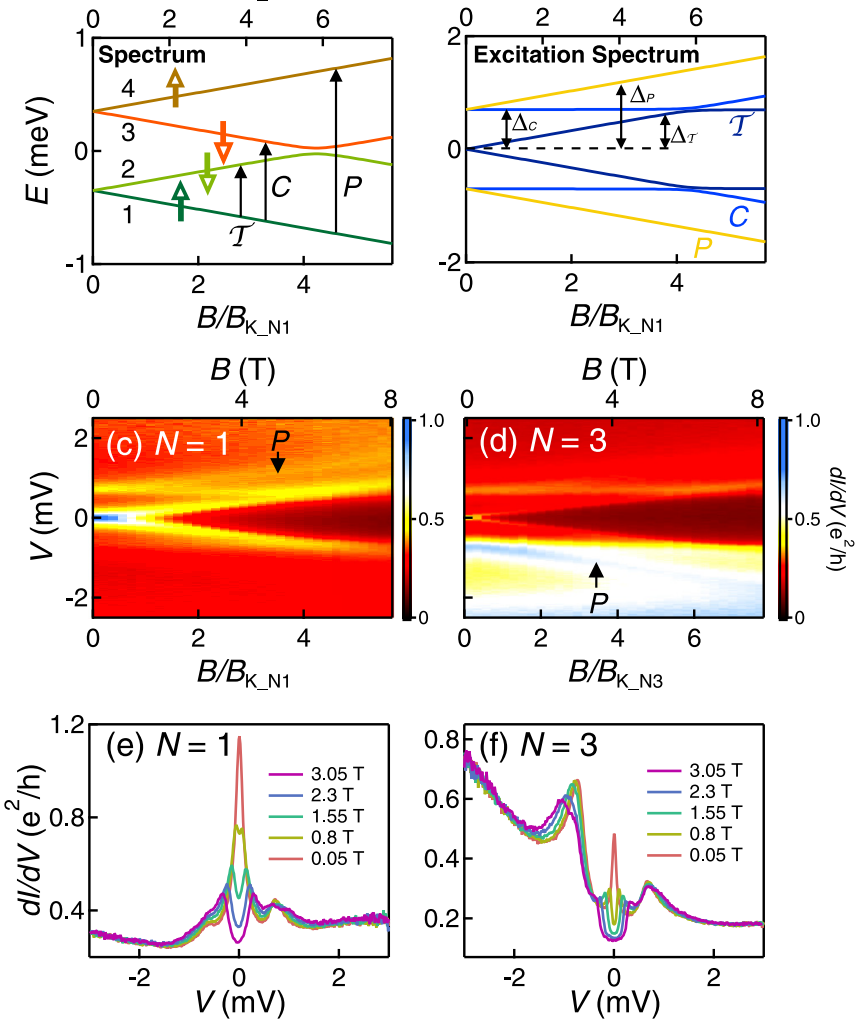

FIG. 2. Behavior in perpendicular magnetic field. (a) Evolution of energy levels in magnetic field; to each level is associated a Kramers pseudospin. (b) Addition spectrum obtained from (a) with inelastic excitations $\Delta_{T}, \Delta_{C}$, and $\Delta_{P}$ associated to $\mathcal{T}, C$, and $P$ transitions being indicated. The magnetic field is scaled by the characteristic fields $B_{K_{N 1}}=1.425 \mathrm{~T}$ and $B_{K_{N 3}}=1.036 \mathrm{~T}$ to emphasize universal behavior. (c), (d) Experimentally measured differential conductance vs scaled magnetic field. The $P$-like resonances are clearly visible and indicated by an arrow. (e), (f) Experimental $d I / d V$ vs bias voltage for various values of the magnetic field for (e) the one-electron and (f) the three-electron valley.

2(d); the reference field $B_{K}=e V_{K} / \mu_{\mathrm{B}}$ is defined through the value $V_{K}$ of the applied bias voltage where the low bias differential conductance $d I / d V$ is $2 / 3$ of its value at zero bias. This ensures universal behavior of the scaled magnetoconductance [22].

Figures 2(e) and 2(f) show $d I / d V$ traces for different values of the magnetic field and characterize the behavior at low fields. We find that the zero-bias peak in valleys $N=1$ and $N=3$ only splits above a critical field of the order of $B_{K}$, as expected from theoretical predictions [23,24]. Further, the inelastic peaks do not split or move at small field values for valley $N=1$, suggesting a predominance of inelastic $C$ transitions [12,13]. Valley $N=3$ can be viewed as a shell with a single hole. Here the side peak at negative bias moves towards larger negative values of the bias voltage as the field increases, suggesting that a $P$-like transition is observed. Hence, the $d I / d V$ is strongly asymmetric in the bias voltage and the $P$-like resonance is seen only for positive (negative) voltages for electron (hole) transport. 
Model and KEA self-energy. To account for this behavior, we have evaluated the $d I / d V$ of a four-level asymmetric Anderson model using the KEA method, which is known to accurately describe the location and height of inelastic Kondo peaks $[12,13]$. The theory, however, becomes imprecise with respect to the width of peaks located at energies much larger than $k_{\mathrm{B}} T_{K}$; hence a comparison with the experiment is only qualitative. The single-particle levels $|j\rangle, j=1, \ldots, 4$ describe a longitudinal mode of a CNT quantum dot with both orbital and spin degrees of freedom [25]. The lower Kramers doublet is the couple $(1,2)$, and the upper the couple $(3,4)$ [see Fig. 2(a). In this basis the interacting CNT Hamiltonian is

$$
\hat{H}_{\mathrm{CNT}}=\sum_{j} \varepsilon_{j}(B) \hat{n}_{j}+\frac{U}{2} \sum_{i \neq j} \hat{n}_{i} \hat{n}_{j}+\hat{H}_{J}
$$

where $\hat{n}_{j}=\hat{d}_{j}^{\dagger} \hat{d}_{j}$ is the occupation operator of level $j$, and $\varepsilon_{1,4}(B)=\varepsilon_{d} \mp \Delta(B) / 2, \varepsilon_{2,3}(B)=\varepsilon_{1,4}(-B)$. Here

$$
\Delta(B)=\sqrt{\Delta_{\mathrm{SO}}^{2}+\left(\Delta_{\mathrm{K}, \mathrm{K}^{\prime}}+g_{s} \mu_{B} B\right)^{2}}=: \Delta_{P}
$$

is the magnetic field dependent level splitting $\left(g_{s} \mu_{B}\right.$ is the spin magnetic moment). Such level splitting yields the addition energy $\Delta_{P}$ for the $P$ resonance. Further, it holds the relation $\Delta_{P}=\Delta_{T}+\Delta_{C}$, with $\Delta_{T}=\varepsilon_{2}-\varepsilon_{1}=\varepsilon_{4}-\varepsilon_{3}$, and $\Delta_{C}=$ $\varepsilon_{3}-\varepsilon_{1}=\varepsilon_{4}-\varepsilon_{2}$. The evolution of the energy levels $\varepsilon_{j}(B)$ in magnetic field is shown in Fig. 2(a). The second and third terms in Eq. (1) account for charging and exchange effects, respectively. We consider strong electron-electron interactions $(U=\infty)$, such that double occupancy of the impurity is excluded and exchange effects are not important.

For a CNT QD defined by electrostatic confinement it can be assumed that the Kramers degrees of freedom are conserved during tunneling [5,17]. Then KEA yields the tunneling density of states (TDOS) of channel $j$ [12],

$$
v_{j}(\varepsilon, B)=\frac{\Gamma / 2 \pi}{\left[\varepsilon_{j}(B)-\varepsilon+\Gamma_{j} \operatorname{Re} \Sigma_{j}(\varepsilon, B)\right]^{2}+\left[\Gamma_{j} \operatorname{Im} \Sigma_{j}(\varepsilon, B)\right]^{2}},
$$

in terms of the KEA self-energies $\Sigma_{j}=\operatorname{Re} \Sigma_{j}+i \operatorname{Im} \Sigma_{j}$ being the central quantities of the theory. Here $\Gamma=\sum_{\alpha, j} \Gamma_{\alpha j} / 4$ is the average coupling and $\Gamma_{\alpha j}$ are the tunneling couplings of channel $j$ at lead $\alpha=L, R$. The current follows from the Meir and Wingreen formula [26]

$$
I=\frac{e}{\hbar} \sum_{j=1}^{4} \int_{-\infty}^{\infty} d \varepsilon \frac{\Gamma_{L j} \Gamma_{R j}}{\Gamma_{L j}+\Gamma_{R j}} v_{j}(\varepsilon)\left[f_{L}(\varepsilon)-f_{R}(\varepsilon)\right],
$$

where $f_{\alpha}=\left[\exp \beta\left(\varepsilon-\mu_{\alpha}\right)+1\right]^{-1}$ is the Fermi function, and $\mu_{L}=\mu_{0}+\eta e V, \mu_{R}=\mu_{0}-(1-\eta)(e V)$ with $\eta \in[0,1]$ accounting for an asymmetric bias drop between the left and right leads. The coupling asymmetry parameter for the lead $\alpha$ and level $j$ is given by $\gamma_{\alpha j}=\Gamma_{\alpha j} / \Gamma_{j}$, with $\Gamma_{j}=\sum_{\alpha} \Gamma_{\alpha j}$.

We keep the SU(2) symmetry within the same Kramers channel, and set

$$
\gamma_{\alpha 1}=\gamma_{\alpha 2}:=\gamma_{\alpha \mathrm{d}}, \quad \gamma_{\alpha 3}=\gamma_{\alpha 4}:=\gamma_{\alpha \mathrm{u}},
$$

as illustrated in Fig. 1(a). Such asymmetries enter in the channel self-energies $\Sigma_{j}$, and hence impact the relevance of a given transition. For occupation $N=1$ we find

$$
\begin{aligned}
\Sigma_{j}(\varepsilon, B)= & \frac{1}{\pi} \sum_{i=T_{j}, C_{j}} \frac{\Gamma_{i}}{\Gamma_{j}}\left[\ln \left(\frac{W}{2 \pi k_{\mathrm{B}} T}\right)+\frac{i \pi}{2}\right. \\
& \left.-\sum_{\alpha} \gamma_{\alpha i} \Psi\left(\frac{1}{2}+\frac{\mathcal{E}}{2 \pi k_{\mathrm{B}} T}-i \frac{\mu_{\alpha}-\varepsilon+\Delta_{j i}}{2 \pi k_{\mathrm{B}} T}\right)\right],
\end{aligned}
$$

where $W$ is a high-energy cutoff, $\Psi$ is the digamma function, $\Delta_{j i}=\varepsilon_{j}-\varepsilon_{i}$, and $T_{j}, C_{j}$ are the $\mathcal{T}$ and $C$ partners of level $j$. Processes which involve the $P$ partner are not explicitly appearing in the KEA self-energies. The case $N=3$ is obtained from Eq. (6) upon replacement of $\Delta_{j i} \rightarrow-\Delta_{j i}$. Finally, the complex quantity $\mathcal{E}$ accounts for low-energy contributions which ensure the proper behavior of the zero-temperature conductance, as discussed in Sec. IV of the Supplemental Material [27].

Impact of asymmetries. The analytic forms Eqs. (3) and (4) allow us to analyze asymmetry effects on the differential conductance $d I / d V$. We focus first on low energies. An expansion of the zero temperature and zero magnetic field differential conductance $G_{\text {diff }}$ in powers of the applied bias, $G_{\text {diff }}=G_{0}+G_{1} V+\ldots$, yields

$$
G_{0}=\frac{2 e^{2}}{h}\left[4 \gamma_{L \mathrm{~d}} \gamma_{R \mathrm{~d}}+\left(\gamma_{L \mathrm{u}} \gamma_{R \mathrm{u}}-\gamma_{L \mathrm{~d}} \gamma_{R \mathrm{~d}}\right) \frac{\pi}{2} \Gamma_{\mathrm{u}} v_{\mathrm{u}}\left(\mu_{0}\right)\right],
$$

being independent of the bias asymmetry $\eta$. The second term within the brackets is proportional to the transmission of the upper Kramers doublet at the Fermi level $\mathcal{T}_{\mathrm{u}}\left(\mu_{0}\right)=$ $\frac{\pi}{2} \Gamma_{\mathrm{u}} v_{\mathrm{u}}\left(\mu_{0}\right)$, and vanishes in the $\mathrm{SU}(4)$ coupling case where $\gamma_{\alpha \mathrm{u}}=\gamma_{\alpha \mathrm{d}}=\gamma_{\alpha}$. Then Eq. (7) yields the known Fermi liquid result $G_{0}=4 \gamma_{L} \gamma_{R} \frac{2 e^{2}}{h}$. The expression for the linear term is lengthier and given in Sec. V of the Supplemental Material. Like $G_{0}, G_{1}$ is also independent of the bias asymmetry $\eta$. Further, it is finite only in the presence of lead asymmetries encapsulated in the parameter $D_{p}=\gamma_{L p}-\gamma_{R p}, p=\mathrm{u}$, d. For finite $D_{\mathrm{u}}=D_{\mathrm{d}}$ and $\Delta=0$ we recover known results for the $\mathrm{SU}(4)$ case [28]. Here the linear term $G_{1}$ is nonvanishing due to a small shift of the TDOS peak from the Fermi energy [29]. These results suggest that the strong asymmetric behavior observed in the experimental data of Fig. 2 requires couplings $\gamma_{\alpha \mathrm{d}} \neq \gamma_{\alpha \mathrm{u}}$.

Resonances at finite bias. We start our analysis by showing in Fig. 3 KEA predictions for $d^{2} I / d V^{2}$. The total linewidth $\Gamma$ is extracted from a fit of the data near the charge peaks, as discussed in Sec. VII of the Supplemental Material. We fix $W / \Gamma=100$; the remaining chosen set of free parameters is shown in Table I. As in the experiment, the KEA currentvoltage characteristics display a $P$ peak at negative (positive) potential drop $e V$ for valley $N=1(N=3)$.

To understand the origin of the resonance, we analyze each individual TDOS $v_{j}$. In general, Kondo resonances appear in the differential conductance when a peak in one or more of the $v_{j}(\varepsilon)$ enters the bias window defined by $\mu_{L}-\mu_{R}=$ $e V$. As seen from Eq. (6), the $v_{j}$ explicitly and significantly depend on the applied bias voltage through their self-energies $\Sigma_{j}$. Further, peaks in $v_{j}$ originate from peaks in $\operatorname{Re} \Sigma_{j}$. At low temperatures, the latter occur when $\varepsilon=\mu_{\alpha}+\Delta_{j i}-\operatorname{Im} \mathcal{E}$, 

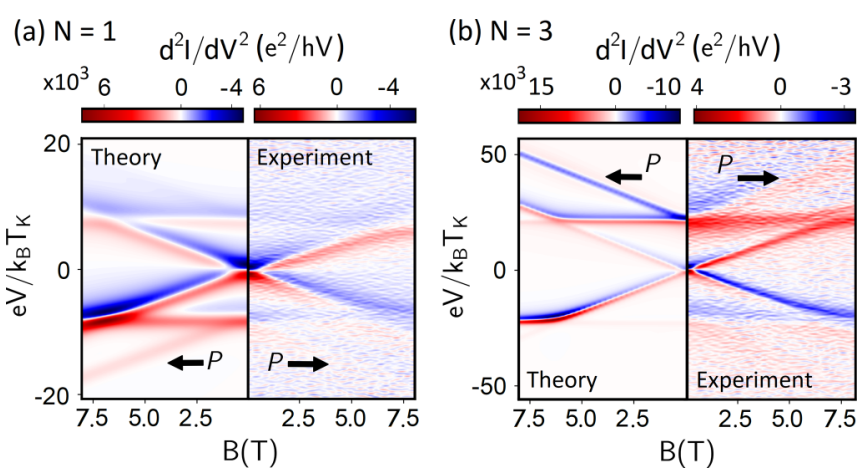

FIG. 3. Second derivative of the current, $d^{2} I / d V^{2}$, for the (a) $N=1$ valley and (b) $N=3$ valley as a function of magnetic field. The left subpanels show analytical predictions for the asymmetric four-level Anderson model, and the right subpanels the experimental observations. Kondo peaks in the differential conductance are manifested as zeros of $d^{2} I / d V^{2}$ as it changes from positive (red) to negative (blue) with increasing bias. Arrows point to the $P$ resonance.

with $i=T_{j}, C_{j}$. Simultaneously, $\operatorname{Im} \Sigma_{j}$ drops by $\gamma_{\alpha i}$ as $\varepsilon$ is swept across the resonance. Conventional Kondo resonances, i.e., the $\mathcal{T}$ and $C$ resonances, arise as a consequence of a peak in $v_{1}$ entering the bias window. The mechanism for the $P$ resonance is different.

In Fig. 4 we focus on the resonance in the $N=1$ valley. We show the energy dependence of each $v_{j}(\varepsilon, e V)$ for different potential drops $e V \simeq \Delta(B)=\Delta_{P}$ for a magnetic field $B=$ $8.05 T$. The gray region indicates the bias window for asymmetric potential drop $\eta=0.42$ and $e V=\Delta_{P}$. From Figs. 4(a) and 4(b), we see that $v_{1}$ is large while $v_{4}$ is negligible in the integration window; further, $v_{1}$ exhibits a monotonic variation as the potential drop increases. Strikingly, $v_{2}$ and $v_{3}$ develop a peak at $e V=\Delta_{P}$ and are of the same order of $v_{1}$, as seen in Figs. 4(c) and 4(d). The peak reflects a resonant feature of $\Sigma_{2}$ and $\Sigma_{3}$, as shown in panels (e) and (f) on the example of $\Sigma_{2}$. This occurs because when $\mu_{L}-\mu_{R}=\Delta_{P}\left(=\Delta_{C}+\Delta_{T}\right)$ the resonances of $\Sigma_{2}\left(\Sigma_{3}\right)$ at $\varepsilon=\mu_{R}+\Delta_{T}$ and $\varepsilon=\mu_{L}-$ $\Delta_{C}\left(\varepsilon=\mu_{R}+\Delta_{C}\right.$ and $\left.\varepsilon=\mu_{L}-\Delta_{T}\right)$ merge into a single concerted resonance.

Correspondingly, the differential magnetoconductance displays a small resonance feature also at bias matching the condition $e V=\Delta_{P}(B)$, as seen in Fig. 3. While the existence of this effect is independent of asymmetries, its magnitude does depend on them. Numerically, for $N=1(N=3)$ we find coupling asymmetry thresholds $\zeta_{1}\left(\zeta_{3}\right)$ above which the resonance is seen. For example, for the valley $N=1$ and $V<0$ it should hold that $\gamma_{L u}-\gamma_{L d}>\zeta_{1}$.

If the coupling strengths are reverted, $\gamma_{L d}-\gamma_{L u}>\zeta_{1}$, the resonance occurs at positive rather than at negative bias. The conditions for the single hole case, $N=3$, can be obtained

TABLE I. Parameters used to fit the experimental data.

\begin{tabular}{cccccc}
\hline \hline$N$ & $\Gamma$ & $\mu_{0}-\varepsilon_{d}$ & $\gamma_{L \mathrm{~d}}$ & $\gamma_{L \mathrm{u}}$ & \multicolumn{1}{c}{$\eta$} \\
\hline 1 & $3.5 \mathrm{meV}$ & $4.83 \Gamma$ & 0.42 & 0.08 & 0.42 \\
3 & $2.4 \mathrm{meV}$ & $5.36 \Gamma$ & 0.3 & 0.06 & 0.1 \\
\hline \hline
\end{tabular}
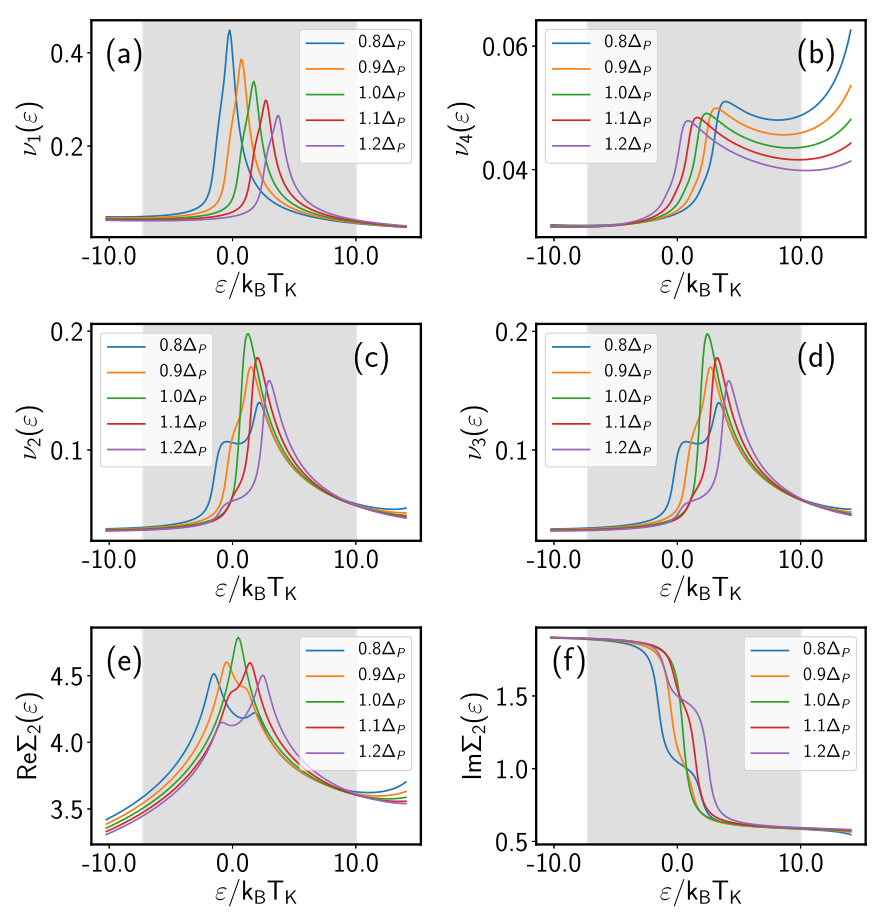

FIG. 4. Channel density of states $v_{j}$, (a)-(d), and self-energy $\Sigma_{2}$ of channel 2, panels (e) and (f), evaluated at bias drops $e V \simeq \Delta_{P}$, where $\Delta_{P}$ is the addition energy of the $P$ resonance, for the $N=1$ case at $B=8.05 T$. The gray stripe indicates the bias window set by the lead chemical potentials when $e V=\Delta_{P}$. At $e V=\Delta_{P}$ the channel density of states $v_{2}$ and $v_{3}$ are maximal and of the order of $v_{1}$. This is due to a resonance of the associated self-energy, as illustrated in (e), (f) on the example of $\Sigma_{2}$. On the other hand, $v_{4}$ is negligible.

from the $N=1$ case by replacing $\mathrm{u} \leftrightarrow \mathrm{d}$ and $\zeta_{1}$ with $\zeta_{3}$. Thus, if a $P$ resonance is observed at positive bias in the $N=1$ valley, it is likely that such resonance also occurs at negative bias in the $N=3$ valley, in agreement with the experiment. Further, bias asymmetries may make it easier to observe a $P$ resonance for small values of $\mu_{0}-\varepsilon_{d}$. The reason is that the tails from the charge-transfer peaks may assist the $P$ peaks if they are located at the same bias polarity. Since the asymmetry parameter $\eta$ defines the bias window for the integration variable $\varepsilon \in\left[\mu_{0}+\eta e V, \mu_{0}-(1-\eta) e V\right], P$ peaks obtained at $\mu_{L}-\mu_{R}>0$ are assisted by charge-transfer tails for $\eta<0.5$. The larger impact of these tails for the $N=3$ valley compared to the $N=1$ one is thus reflected in different asymmetries $\eta$ (see Table I).

Conclusion. We have observed the emergence of inelastic resonances at bias voltages corresponding to pseudospin conserving $P$ transitions in a Kondo-correlated CNT-QD. These resonances emerge nontrivially from a coherent addition of pseudospin nonconserving $\mathcal{T}$ and $C$ transitions. The here established mechanism for $P$-like resonance becomes prominent in the presence of asymmetries in the tunneling coupling and bias drop.

Acknowledgments. We acknowledge support by the Deutsche Forschungsgemeinschaft (DFG) Project ID 314695032-SFB 1277 B04, and by JSPS KAKENHI Grants No. JP15J01518, No. JP19H00656, and No. JP19H05826.

A.L. and T.H. contributed equally to this work. 
[1] J. Kondo, Prog. Theor. Phys. 32, 37 (1964).

[2] D. Goldhaber-Gordon, H. Shtrikman, D. Mahalu, D. AbuschMagder, U. Meirav, and M. A. Kastner, Nature (London) 391, 156 (1998).

[3] S. M. Cronenwett, T. H. Oosterkamp, and L. P. Kouwenhoven, Science 281, 540 (1998).

[4] P. Jarillo-Herrero, J. Kong, H. S. J. van der Zant, C. Dekker, L. P. Kouwenhoven, and S. De Franceschi, Nature (London) 434, 484 (2005).

[5] M.-S. Choi, R. López, and R. Aguado, Phys. Rev. Lett. 95, 067204 (2005).

[6] A. Makarovski, J. Liu, and G. Finkelstein, Phys. Rev. Lett. 99, 066801 (2007).

[7] F. B. Anders, D. E. Logan, M. R. Galpin, and G. Finkelstein, Phys. Rev. Lett. 100, 086809 (2008).

[8] M. Ferrier, T. Arakawa, T. Hata, R. Fujiwara, R. Delagrange, R. Weil, R. Deblock, R. Sakano, A. Oguri, and K. Kobayashi, Nat. Phys. 12, 230 (2016).

[9] M. Ferrier, T. Arakawa, T. Hata, R. Fujiwara, R. Delagrange, R. Deblock, Y. Teratani, R. Sakano, A. Oguri, and K. Kobayashi, Phys. Rev. Lett. 118, 196803 (2017).

[10] T.-F. Fang, W. Zuo, and H.-G. Luo, Phys. Rev. Lett. 101, 246805 (2008).

[11] J. P. Cleuziou, N. V. N'Guyen, S. Florens, and W. Wernsdorfer, Phys. Rev. Lett. 111, 136803 (2013).

[12] D. R. Schmid, S. Smirnov, M. Margańska, A. Dirnaichner, P. L. Stiller, M. Grifoni, A. K. Hüttel, and C. Strunk, Phys. Rev. B 91, 155435 (2015).

[13] M. Niklas, S. Smirnov, D. Mantelli, M. Marganska, N.-V. Nguyen, W. Wernsdorfer, J.-P. Cleuziou, and M. Grifoni, Nat. Commun. 7, 12442 (2016).

[14] M. R. Galpin, F. W. Jayatilaka, D. E. Logan, and F. B. Anders, Phys. Rev. B 81, 075437 (2010).

[15] D. Mantelli, C. Moca, G. Zaránd, and M. Grifoni, Physica E 77, 180 (2016).

[16] J. R. Schrieffer and P. A. Wolff, Phys. Rev. 149, 491 (1966).

[17] J. S. Lim, M.-S. Choi, M. Y. Choi, R. López, and R. Aguado, Phys. Rev. B 74, 205119 (2006).

[18] J. Nygård, D. H. Cobden, and P. E. Lindelof, Nature (London) 408, 342 (2000).

[19] P. Jarillo-Herrero, J. Kong, H. S. J. van der Zant, C. Dekker, L. P. Kouwenhoven, and S. De Franceschi, Phys. Rev. Lett. 94, 156802 (2005).
[20] T. Jespersen, K. Grove-Rasmussen, J. Paaske, K. Muraki, J. Nygård, and K. Flensberg, Nat. Phys. 7, 348 (2011).

[21] S. Smirnov and M. Grifoni, Phys. Rev. B 87, 121302(R) (2013).

[22] M. Gaass, A. K. Hüttel, K. Kang, I. Weymann, J. von Delft, and C. Strunk, Phys. Rev. Lett. 107, 176808 (2011).

[23] T. Costi, Phys. Rev. Lett. 85, 1504 (2000).

[24] S. Smirnov and M. Grifoni, New J. Phys. 15, 073047 (2013).

[25] E. A. Laird, F. Kuemmeth, G. A. Steele, K. Grove-Rasmussen, J. Nygård, K. Flensberg, and L. P. Kouwenhoven, Rev. Mod. Phys. 87, 703 (2015).

[26] Y. Meir and N. S. Wingreen, Phys. Rev. Lett. 68, 2512 (1992).

[27] See Supplemental Material at http://link.aps.org/supplemental/ 10.1103/PhysRevB.101.041102 for detailed discussion of the model Hamiltonian, tunneling density of states in the Keldysh effective action, conditions for unitary conductance, low bias expansion of the differential conductance, TDOS and self-energy signatures of Kondo transitions, and matching between the theory and the experiment, which includes Refs. [5,12,15,21,26,28-38].

[28] C. Mora, P. Vitushinsky, X. Leyronas, A. A. Clerk, and K. Le Hur, Phys. Rev. B 80, 155322 (2009).

[29] A. C. Hewson, The Kondo Problem to Heavy Fermions, Cambridge Studies in Magnetism (Cambridge University Press, Cambridge, UK, 1993).

[30] Y. Meir, N. S. Wingreen, and P. A. Lee, Phys. Rev. Lett. 70, 2601 (1993).

[31] N. S. Wingreen and Y. Meir, Phys. Rev. B 49, 11040 (1994).

[32] A. Kamenev, Field Theory of Non-Equilibrium Systems (Cambridge University Press, Cambridge, UK, 2011).

[33] A. Altland and B. Simons, Condensed Matter Field Theory (Cambridge University Press, Cambridge, UK, 2006).

[34] D. C. Langreth, Phys. Rev. 150, 516 (1966).

[35] R. Sakano and N. Kawakami, Phys. Rev. B 73, 155332 (2006).

[36] S. Smirnov and M. Grifoni, Phys. Rev. B 84, 125303 (2011).

[37] M. Pletyukhov and H. Schoeller, Phys. Rev. Lett. 108, 260601 (2012).

[38] C. W. J. Beenakker, Phys. Rev. B 44, 1646 (1991). 